\title{
IMPACT ON SOME PLANNING DECISIONS FROM A FUZZY MODELLING
}

OF POWER SYSTEMS

\author{
J. Tomé Saraiva \\ Vladimiro Miranda \\ INESC - Instituto de Engenharia de Sistemas e Computadores \\ and FEUP/DEEC - Faculdade de Engenharia da Universidade do Porto \\ L. Mompilher - 4000 Porto - PORTUGAL \\ Fax: 351.2.318692 ean: v_miranda@inescn.pt
}

\author{
L.M.V.G. Pinto \\ PUC/RJ - Pontifícia Universidade Católica \\ Rio de Janeiro \\ C.P. 38063 Gávea - CEP 22452 RJ \\ BRASIL
}

Abstract - In this paper, system component reinforcements are analyzed from the perspective of their impact in increasing flexibility in system design. The proposed framework integrates a fuzzy optimal power flow model through which one can derive, as a function of load uncertainties, possibility distributions for generation, power flows and power not supplied. Exposure and robustness indices, based on risk analysis concepts, are defined. These indices can be used to rank the expansion alternatives, giving the planner insight to system behavior in face of adverse futures. Their use in conjunction with investment assessments is proposed as a necessary step in a decision making methodology.

\section{INTRODUCTION}

When planning a power system expansion, several types of uncertainties must be dealt with. Some of these, such as the ones related with system or component failures, are adequately taken care of by probabilistic methods and their impact assessed by reliability studies. But other, such as in load forecast, cannot be represented by a probabilistic approach: not only many of the underlying assumptions are of qualitative nature but also unlikely events may have catastrophic consequences and planners wish to take that into account. Moreover, planners tend to search for some sort of "flexible planning" instead of for some kind of special solution, that may be "optimal" for the expectancy of some variable but prove disastrous if the future does not come as expected (which usually happens...).

In this paper one defines nodal consumptions as fuzzy loads, associated to a possibility distribution, using concepts from the fuzzy set theory $[1,2,3]$. This means that one has an imprecise definition, covering a range of values depending on the nature of the uncertainty about the exact value. This imprecise definition is distinct from a probabilistic approach, which is seen as misadjusted, because in planning exercises it seldom makes real sense to adjust any kind of probability distribution to load values; besides, one will be trying to find "what may happen" in the future, and will not be searching for any kind of expectancy.

Having loads as uncertain defines the problem within a domain of possible future load scenarios. The purpose of this paper is to show that decisions on setting or reinforcing system component capacities (such as in generation or line power flow) may be evaluated in terms of how much or how well that domain of possibilities is covered. For this purpose, robustness and exposure indices are defined, characterizing the risk of having taken good or regrettable decisions concerning the system ability to cope with uncertainty. These indices may be used to compare alternative designs, to be set as design targets or to rank system reinforcement strategies so that a plan may be selected not only on the basis of economic information related to investments, but also on the ability of each alternative to accommodate imprecision in load values.

In fact, some expensive investments may show to have virtually no impact in introducing flexibility in system design, while others may prove to have a definite influence for such purpose.

If loads are uncertain, then generations (including optimal generations) and lines flows will be uncertain as well. The Fuzzy Power Flow problem has already been addressed, in DC and AC formulations $[3,4,5]$. But, as generations are not given as data in a planning environment, the above mentioned problem must instead be solved within the framework of the Fuzzy Optimal Power Flow (FOPF) approach. A first FOPF model has been presented in [6]; however, it could not deal adequately with some possible extreme situations and, therefore, its results were in some cases optimistic (in the sense that the possibility distributions for generations and line flows, resulting from its application, could be too narrow).

In this paper one presents a new FOPF model, organized as a multiparametric programming formulation. As both the new algorithm proposed and the operations with fuzzy sets are very efficient, from a computational point of view, a computer application could be developed suited to be used as frequently as needed and even to be integrated in more complex procedures (such as a Monte Carlo, e.g.).

In order to illustrate the proposed techniques and the use of the proposed indices, some results are presented based on studies on the IEEE 24 bus Reliability Test System.

\section{ABOUT FUZZY SET THEORY}

A fuzzy set $\tilde{\mathrm{A}}$ is characterized by a membership function $\mu \tilde{\mathrm{A}}(\mathrm{x})$ relating each element $\mathrm{x}_{1}$ to its compatibility degree with $\mathrm{X}_{1}$. In this case, the transition between the extreme situations of full and complete lack of membership is gradual. In certain circumstances, a fuzzy set can be associated to a possibility distribution. In such cases, the membership function corresponds to a possibility distribution function.

$$
\tilde{\mathrm{A}}=\left\{\left(\mathrm{x}_{1}, \mu \tilde{\mathrm{A}}\left(\mathrm{x}_{1}\right)\right), \mathrm{x}_{1} \in \mathrm{X}_{1}\right\}
$$

An $\alpha$-level set or an $\alpha$-cut of a fuzzy set $\tilde{A}$ defined in $X_{1}$ is the hard set $\mathrm{A}_{\alpha}$ obtained from $\tilde{\mathrm{A}}$ for each $\alpha \in[0,1]$ such that:

$$
\mathrm{A}_{\alpha}=\left\{\mathrm{x}_{1} \in \mathrm{X}_{1}: \mu \tilde{\mathrm{A}}\left(\mathrm{x}_{1}\right) \geq \alpha\right\}
$$

The support set of a fuzzy set $\tilde{A}, S(\tilde{A})$, is the hard set containing the elements of $X_{1}$ having their membership function in ]0,1]. A fuzzy set $\tilde{A}$ is said to be a fuzzy number if it is a convex fuzzy set of the real line $\mathrm{R}$ such that its membership function is piecewise continuous. The usual set operations can be readily extended to fuzzy sets using the Extension Principle formulated by Zadeh. In particular, given the fuzzy sets $\tilde{A}$ and $\tilde{O}$, where $\tilde{O}$ is

$$
\tilde{\mathrm{O}}=\left\{\left(\mathrm{y}_{1}, \mu \tilde{\mathrm{O}}\left(\mathrm{y}_{1}\right)\right), \mathrm{y}_{1} \in \mathrm{X}_{1}\right\}
$$




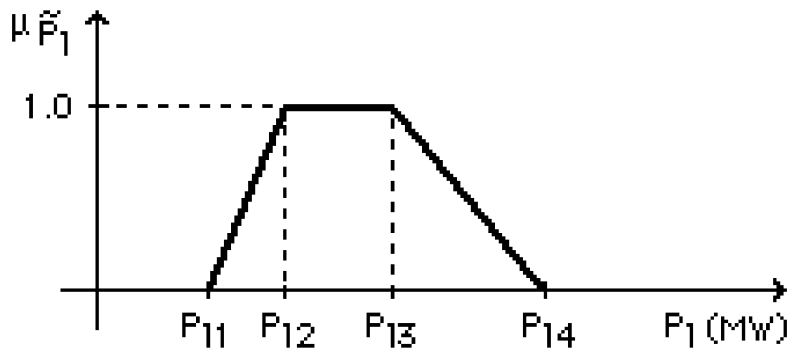

Fig.1 - Trapezoidal fuzzy number modelling a fuzzy load.

the union ÃUÕ is calculated by

$$
\begin{aligned}
& \tilde{\mathrm{A} U O \tilde{O}}=\left\{\left(\mathrm{z}_{1}, \mu \tilde{\mathrm{A} U} \mathrm{O}\left(\mathrm{z}_{1}\right)\right), \mathrm{z}_{1} \in \mathrm{X}_{1}\right\} \\
& \mu \tilde{\mathrm{A} U O \tilde{O}}\left(\mathrm{z}_{1}\right)=\max \left\{\mu \tilde{\mathrm{A}}\left(\mathrm{z}_{1}\right), \mu \tilde{O}\left(\mathrm{z}_{1}\right)\right\}
\end{aligned}
$$

It should also be emphasized that the convolution used to extend several arithmetic operations to fuzzy sets is much simpler than if probabilistic concepts are considered. This fact strongly contributes to make arithmetic operations with fuzzy sets very efficient in a computational framework.

\section{DEFINITION OF FUZZY LOADS}

The modelization and specification of fuzzy loads has been addressed in [3]. In this paper, trapezoidal fuzzy numbers (figure 1) associated to a membership function are used to represent a vague knowledge about the load behavior.

The planning engineer is supposed to declare an interval $\left[\mathrm{P}_{12}\right.$, $\mathrm{P}_{13}$ ] integrating a set of values having high credibility, being considered as good representations of load characteristics. Besides, values $\mathrm{P}_{11}$ and $\mathrm{P}_{14}$, under and above which the load is considered not possible to occur, are also be specified. Then, values in the intervals $\left[\mathrm{P}_{11}, \mathrm{P}_{12}[\right.$ and ] $\left.\mathrm{P}_{13}, \mathrm{P}_{14}\right]$ are taken as possible representations of the load but not with the same strength as values in $\left[\mathrm{P}_{12}, \mathrm{P}_{13}\right]$. This representation can be considered as the translation of the following linguistic declaration (see figure 1): load may occur between $P_{l 1}$ and $P_{l 4}$ but it is likely to occur in $\left[P_{l 2}, P_{l 3}\right]$.

Fuzzy numbers are a very convenient framework to integrate vague information in models: performing unary and binary arithmetic operations is based on very fast formulas that nevertheless provide exact results. This feature strongly contributes to develop high performance algorithms and, in what power systems are concerned, to access network behavior information in a decision aid framework.

\section{FUZZY DC OPTIMAL POWER FLOW MODEL}

\section{$\underline{\text { General description }}$}

A FOPF methodology is an optimization procedure to identify generation membership functions driven by an economic criterion under uncertain loads. The developed methodology adopts a DC formulation integrating the active power balance equation and generation and branch power flow limit constraints. The DC model is adopted as the problem addressed is of planning.

In figure 2 the FOPF algorithm is presented. It starts by a deterministic DC optimal power flow so that an optimal and feasible solution related to the central values of the $1.0 \alpha$-cut of the specified load membership functions is identified.

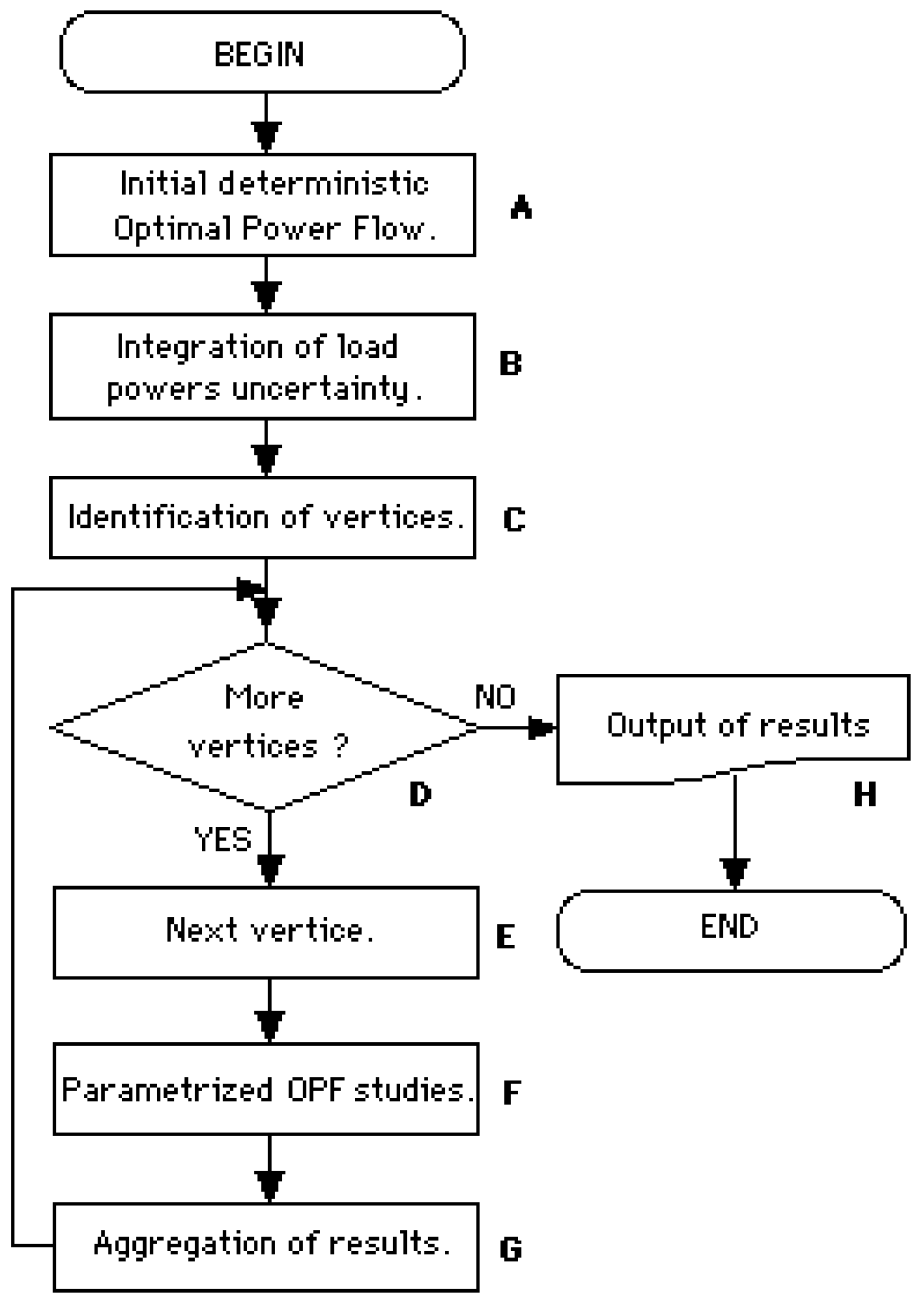

Fig. 2 - Fuzzy DC Optimal Power Flow Algorithm.

The generator cost functions are linearized in the usual way namely for those having non linear convex cost functions (decomposed in two or more linear sections according to their nonlinearity degree). In other words, these generators are represented by a number of fictitious generators having constant incremental costs. Load imprecisions are integrated in the optimization problem by adopting a parametric representation of the $0.0 \alpha$-cut of each load membership function .

This way a multiparametric optimization problem is formulated. Gal [7] presents an algorithm to identify regions related to feasible and optimal solutions. This algorithm is considered to have rather poor computational performances if real sized problems are to be dealt with. Therefore, a new and efficient algorithm was developed to solve, even if in a approximate way, the previous problem.

The proposed algorithm identifies vertices of the hypervolume enclosing the possible load values and analyses each one by performing parametric linear programming studies. The generator, branch power flows and power not supplied membership functions are finally obtained by aggregating the solutions of these parametric studies, using fuzzy operators.

\section{$\underline{\text { Initial Deterministic Optimal Power Flow }}$}

The initial deterministic OPF model is given by equations (6) to (12). In this model $\mathrm{P}_{\text {gi }}$ and $\mathrm{PNS}_{\mathrm{i}}$ represent, respectively, the active power at bus $i$ generator and the power not supplied at bus $i$.

The coefficient $c_{i}$ represents the incremental cost of the generator connected to bus $i$ and $G$ is the marginal cost of the fictitious 
generators related to the active power not supplied. Constraints (8) and (9) are related to the generation power limits at bus i while (10) represents the maximum power not supplied at bus $i$.

This limit, $\mathrm{P}_{\text {liav }}$, corresponds to the average value of $1.0 \alpha$-cut of bus i load membership function. Constraints (11) and (12) are related to the limits of the power flow in branch $\mathrm{j}$. In these constraints $a_{j i}$ represents the DC model sensitivity coefficient relating the branch $j$ power flow to the bus i active injected power.

$$
\begin{aligned}
\min \mathrm{z}= & \sum \mathrm{c}_{\mathrm{i}} \cdot \mathrm{P}_{\text {gi }}+\mathrm{G} \cdot \sum \mathrm{PNS}_{\mathrm{i}} \\
\text { subj. } & \sum \mathrm{P}_{\text {gi }}+\sum \mathrm{PNS}_{\mathrm{i}}=\sum \mathrm{P}_{\text {liav }} \\
& \mathrm{P}_{\text {gi }} \leq \mathrm{P}_{\text {gimax }} \\
& \mathrm{P}_{\text {gi }} \geq \mathrm{P}_{\text {gimin }} \\
& \mathrm{PNS}_{\mathrm{i}} \leq \mathrm{P}_{\text {liav }} \\
& \sum \mathrm{a}_{\mathrm{ji}} \cdot\left(\mathrm{P}_{\text {gi }}-\mathrm{P}_{\text {liav }}+\mathrm{PNS}_{\mathrm{i}}\right) \leq \mathrm{P}_{\mathrm{jmax}} \\
& \sum \mathrm{a}_{\mathrm{ji}} \cdot\left(\mathrm{P}_{\text {gi }}-\mathrm{P}_{\text {liav }}+\mathrm{PNS}_{\mathrm{i}}\right) \geq \mathrm{P}_{\mathrm{jmin}}
\end{aligned}
$$

\section{$\underline{\text { Integration of load uncertainties }}$}

Load uncertainties associated to the $0.0 \alpha$-cut can be considered by integrating, in the previous problem, parameters $\Delta_{i}$ (13) related to each load membership function. The active power balance equation and the branch flow limit constraints considering these parameters are now given, respectively, by (14) to (16).

$$
\begin{aligned}
& \Delta_{\mathrm{i}}=\mathrm{P}_{\mathrm{li}}-\mathrm{P}_{\text {liav }} \\
& \sum \mathrm{P}_{\mathrm{gi}}+\sum \mathrm{PNS}_{\mathrm{i}}=\sum\left(\mathrm{P}_{\text {liav }}+\Delta_{\mathrm{i}}\right) \\
& \sum \mathrm{a}_{\mathrm{ji}} \cdot\left(\mathrm{P}_{\text {gi }}-\mathrm{P}_{\text {liav }}+\mathrm{PNS}_{\mathrm{i}}\right) \leq \mathrm{P}_{\mathrm{jmax}}+\sum \mathrm{a}_{\mathrm{ji}} \Delta_{\mathrm{i}} \\
& \sum \mathrm{a}_{\mathrm{ji}} \cdot\left(\mathrm{P}_{\mathrm{gi}}-\mathrm{P}_{\text {liav }}+\mathrm{PNS}_{\mathrm{i}}\right) \geq \mathrm{P}_{\mathrm{jmin}}+\sum \mathrm{a}_{\mathrm{ji}} \Delta_{\mathrm{i}}
\end{aligned}
$$

The FOPF problem including information on load uncertainties can be formulated as a multiparametric linear programming problem, given by equations (17) to (19), where $\mathrm{A}$ is the coefficient matrix, $\mathrm{X}$ is the vector of variables, $\mathrm{b}$ is the right hand side vector, $\mathrm{b}^{\prime}(\Delta)$ is a vector of linear expressions on $\Delta \mathrm{s}$ :

$$
\begin{aligned}
& \min z=c^{\mathrm{t}} \cdot \mathrm{X} \\
& \text { subj. } \quad \text { A.X }=\mathrm{b}+\mathrm{b}^{\prime}(\Delta) \\
& \Delta_{\text {imin }} \leq \Delta_{\mathrm{i}} \leq \Delta_{\text {imax }} \\
& \mathrm{b}^{\prime}(\Delta) \text { is: } \quad \sum \Delta_{\mathrm{i}}, \quad \text { for type (14) constraint } \\
& \sum \mathrm{a}_{\mathrm{ji}} \Delta_{\mathrm{i}} \quad \text { for type (15) constraints } \\
& -\sum \mathrm{a}_{\mathrm{ji}} \Delta_{\mathrm{i}} \quad \text { for type (16) constraints }
\end{aligned}
$$

This leads to the identification of the $\Delta$ values associated to the extreme values of each of the basic optimal variables. The inclusion of the $\Delta$ parameters allows the optimal solution of (6-12) to be described as a set of linear expressions on $\Delta$ :

$$
\mathrm{X}^{\mathrm{opt}}=\mathrm{B}^{-1}\left(\mathrm{~b}+\mathrm{b}^{\prime}(\Delta)\right)
$$

\section{Identification of vertices}

From the hypervolume defined by (19), representing the domain of uncertain loads, two kinds of vertices are interesting to look for:

a) Feasible vertices that lead to maxima or minima of the basic variables of the optimal solution of problem (6-12), when solved with (17-19).

b) Unfeasible vertices for the basic variables set in (20).

The set of $\Delta_{\mathrm{i}}$ values that minimizes and maximizes each variable is readily identified. For instance, if the maximum value of $x_{i}$ is to be obtained, $\Delta_{\text {imax }}$ should be considered whenever the $\Delta_{\mathrm{i}}$ coefficient in the right hand side of (20) is positive; $\Delta_{\text {imin }}$ should instead be selected if that coefficient is negative. This set of $\Delta_{\mathrm{i}}$ values corresponds to a vertex of the hipervolume as, for each $\Delta_{\mathrm{i}}$, this vertex is only defined by $\Delta_{\mathrm{imin}}$ or $\Delta_{\mathrm{imax}}$ values. For this set of $\Delta_{\mathrm{i}}$ values, the other basic variable values can be calculated. If the value of, at least, one of these is negative, then the initial optimal solution is not feasible in a region including the identified vertex.

This procedure is done for all the basic variables, resulting in the identification of all the vertices, surrounding the initial central value, that lead to individual extreme values of the basic variables.

\section{$\underline{\text { Parametric OPF problems }}$}

Let us consider a power system supplying two loads ( $\mathrm{P}_{11}$ and $P_{12}$ ) represented by trapezoidal fuzzy numbers (21) and (22). The load uncertainties can be expressed in terms of $\Delta 1$ and $\Delta 2$ by (23) and (24). In figure 3 the rectangles corresponding to the 0.0 and $1.0 \alpha$-cuts are represented in terms of $\Delta 1$ and $\Delta 2$.

$$
\begin{aligned}
& \mathrm{P}_{11}=\left(\mathrm{P}_{11} ; \mathrm{P}_{112} ; \mathrm{P}_{113} ; \mathrm{P}_{114}\right) \\
& \mathrm{P}_{12}=\left(\mathrm{P}_{12} ; \mathrm{P}_{12} ; \mathrm{P}_{123} ; \mathrm{P}_{124}\right) \\
& \mathrm{P}_{11}=\mathrm{P}_{112}+\left(\Delta_{11} ; \Delta_{12} ; \Delta_{13} ; \Delta_{14}\right) \\
& \mathrm{P}_{12}=\mathrm{P}_{122}+\left(\Delta_{21} ; \Delta_{22} ; \Delta_{23} ; \Delta_{24}\right)
\end{aligned}
$$

After a vertex $\mathrm{Y}$ has been identified in the previous step, a linear parametric model is adopted. Referring to fig. 3, load sets in segments $\mathrm{OX}$ and $\mathrm{XY}$ can be considered by solving two parametric linear programming problems in a successive way. Starting at the initial optimal solution for point $\mathrm{O}\left(\Delta_{1}=0.0\right.$ and $\left.\Delta_{2}=0.0\right)$ the first parametric problem can be solved by associating $\partial=1.0$ to point $\mathrm{O}$ and $\partial=0.0$ to point $\mathrm{X}$. The second parametric study can be performed reseting the $\partial$ parameter to 1.0 and starting at the feasible optimal solution related to $\mathrm{X}$. Whenever a change of optimal basis is required, in order to keep feasibility, the Dual Simplex algorithm is used so that new feasible and optimal basis (while $\partial$ varies in the interval $[0,1]$ ) may be identified, in each stage. Moreover, the $\partial$ parameter can be identified with $\alpha$-cut levels in the second stage.

Let us consider the analysis of loads over a general segment: for instance, $\mathrm{XY}$ (the procedure for $\mathrm{OX}$ is identical). If $\partial=1$ is related to $\Delta \mathrm{X}$ and $\partial=0$ to $\Delta \mathrm{Y}$ then the power balance equation and the branch power flow limits are, respectively, given by (25) to (27).

$$
\begin{aligned}
\sum \mathrm{P}_{\text {gi }}+\sum \mathrm{PNS}_{\mathrm{i}}=\sum\left(\mathrm{P}_{\text {liav }}\right. & \left.+\Delta \mathrm{X}_{\mathrm{i}}+\left(1.0-\partial_{\mathrm{i}}\right)\left(\Delta \mathrm{Y}_{\mathrm{i}}-\Delta \mathrm{X}_{\mathrm{i}}\right)\right) \\
\sum \mathrm{a}_{\mathrm{ji}}\left(\mathrm{P}_{\text {gi }}-\mathrm{P}_{\text {liav }}-\Delta \mathrm{X}_{\mathrm{i}}+\mathrm{PNS}_{\mathrm{i}}\right) \leq \mathrm{P}_{\mathrm{jmax}}+ & + \\
& +\sum \mathrm{a}_{\mathrm{ji}}\left(1.0-\partial_{\mathrm{i}}\right)\left(\Delta \mathrm{Y}_{\mathrm{i}}-\Delta \mathrm{X}_{\mathrm{i}}\right) \\
\sum \mathrm{a}_{\mathrm{ji}}\left(\mathrm{P}_{\text {gi }}-\mathrm{P}_{\text {liav }}-\Delta \mathrm{X}_{\mathrm{i}}+\right. & \left.\mathrm{PNS}_{\mathrm{i}}\right) \geq \mathrm{P}_{\mathrm{jmin}}+ \\
& +\sum \mathrm{a}_{\mathrm{ji}}\left(1.0-\partial_{\mathrm{i}}\right)\left(\Delta \mathrm{Y}_{\mathrm{i}}-\Delta \mathrm{X}_{\mathrm{i}}\right)
\end{aligned}
$$

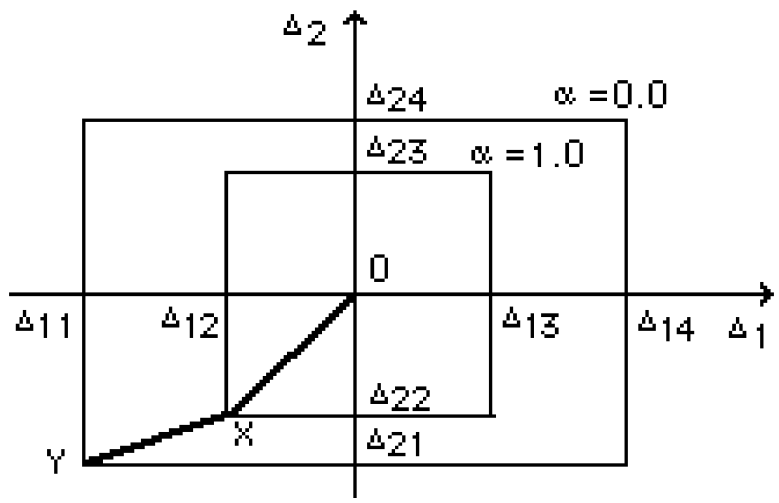


Fig. 3 - Rectangles corresponding to 0.0 and $1.0 \alpha$-cuts of load membership functions for two loads.

The first parametric study is performed considering that points $\mathrm{O}$ and $\mathrm{X}$ correspond, respectively, to $\partial=1.0$ and $\partial=0.0$ so that problem (28) to (30) is obtained.

$$
\begin{array}{ll}
\min z= & c^{t} \cdot X \\
\text { subj. } & \text { A.X }=b+b^{\prime}(1.0-\partial) \\
& 0.0 \leq \partial \leq 1.0
\end{array}
$$

When point $\mathrm{X}$ is reached, i.e., a feasible and optimal solution to $\partial=0.0$ is identified, the optimal feasible solution is given by (31). where $\mathrm{B}$ is the base matrix, that is, the matrix having the columns of the basic variables in problem (28) to (30).

$$
\mathrm{Xopt}=\mathrm{B}-1 .\left(\mathrm{b}+\mathrm{b}^{\prime}(1.0-\partial)\right)
$$

In the second parametric study, points $\mathrm{X}$ and $\mathrm{Y}$ correspond to $\partial=1.0$ and $\partial=0.0$, so that the previous optimal solution in $X$ is now given by (30). In expressions (31) and (32), $b^{\prime}$ and $b^{\prime \prime}$ represent the terms depending from $\partial$ in the two parametric problems. $\mathrm{B}$ is the base matrix associated to the optimal and feasible solution related to point $\mathrm{X}$ of figure 3 .

$$
\mathrm{Xopt}=\mathrm{B}-1 .\left(\mathrm{b}+\mathrm{b}^{\prime}+\mathrm{b}^{\prime \prime}(1.0-\partial)\right)
$$

As a result of each pair of parametric programming problems, a set of optimal values, associated to a possibility degree, is obtained for each variable. In figures 4.a) b) and c) three possible representations of these solutions are presented. In figures 4.a) to c) break points A, B, C and $\mathrm{D}$ correspond to $\partial$ values for which the current optimal basis became unfeasible and so an iteration of the Dual Simplex Method had to be performed. The set of solutions presented in figure 4.c) must be transformed into a possibility distribution using the Max operator so that the distribution sketched in figure 5 can be obtained.

Fig. 4 - Three hypothetical trajectories describing the change in a variable Xi with decreasing membership values.

Fig. 5 - Possibility distribution obtained from fig. 4.c) by applying the Max operator.

\section{Aggregation of results}

The generated active power and branch flow possibility distributions obtained with each parametric programming exercise must finally be aggregated using the fuzzy union operator. This way, correct possibility distributions, integrating only possible optimal generated or branch flow power values, can be built.

The final results from a FOPF exercise are possibility distributions for:
a) generated powers;
b) line and transformer power flows;
c) system power not supplied.

\section{ROBUSTNESS AND EXPOSURE INDICES}

The FOPF model allows one to detect for which set of lumped and nested load scenarios the system will have feasible solutions, while keeping track of the more economic ones. This set can be designated as a set for whose uncertainties (in whatever way they may be combined) the system is robust. This is a concept present in risk analysis, whose general ideas were well summarized in [8].

A robustness index can be associated with this set: it just takes the value (1- ) where is the lowest -cut level for which the system still accommodates data uncertainties at that level. An exposure index is also readily available, taking the value , and is associated with at least one system component (generation or transmission) that creates a bottleneck in system flexibility to accommodate uncertainties in data. The exposure index can also be interpreted as the highest -cut of the PNS possibility distribution having non-zero values. In figure 6 an example of such a possibility distribution is sketched. According to this representation the system exposure index is 1. This means that the system can accommodate the specified uncertainties regarding the levels from 1.0 to 1 . If lower -cuts are analyzed, that is if the imprecision grows up, the power not supplied assumes non zero values. This way the exposure and robustness indices are 1 and 1.0- 1 .

\section{REINFORCEMENT STRATEGY IDENTIFICATION}

Among the results obtained with the FOPF methodology one gets a distribution for the power not supplied. The PNS non-zero values depend on system components originating bottlenecks in the system capacity to accommodate uncertainty. Therefore, the risk in keeping the system as it is, in face of the uncertainties in future loads, may be reduced if component reinforcements are specified. The identification of expansion plans can be considered in two complementary ways:

a) the planner may specify reinforcements and evaluate their impact on the exposure and robustness indices;

Fig. 6 - Example of a PNS possibility distribution.

b) the planner may specify target values for the exposure (or robustness) index and indicate those generators or branches whose limits may be modified; the reinforcement values for the specified components must then be calculated.

The evaluation of the exposure and robustness indices within the first option can be simply done by solving a new FOPF considering the specified reinforcement values.

In the second option, an expansion plan can be built by adding variables related to each component to be reinforced in several phases of the FOPF methodology. Let us consider variables $\Delta \mathrm{Pgi}$ and $\Delta \mathrm{Pj}$, related to generator $\mathrm{i}$ and branch $\mathrm{j}$ power limit increases. Constraints (33) to (35) are related to the initial deterministic optimal power flow problem and correspond to the generator $i$ active power limit and to the minimum and maximum values of the branch $\mathrm{j}$ active power flow.

$$
\begin{aligned}
& \text { Pgi } \leq \text { Pgimax }+\Delta \text { Pgi } \\
& \sum \text { aji }(\text { Pgi }- \text { Pliav }+ \text { PNSi }) \leq \text { Pjmax }+\Delta P j \\
& \sum \text { aji }(\text { Pgi }- \text { Pliav }+ \text { PNSi }) \geq P j m i n-\Delta P j
\end{aligned}
$$

If the parametric optimal power flows are considered, constraints related to branch power flow are given by (36) and (37). In this case, constraint (33) remains unchanged.

$$
\begin{array}{r}
\sum \mathrm{a}_{\mathrm{ji}}\left(\mathrm{P}_{\text {gi }}-\mathrm{P}_{\text {liav }}-\Delta \mathrm{X}_{\mathrm{i}}+\mathrm{PNS}_{\mathrm{i}}\right) \leq \mathrm{P}_{\mathrm{jmax}}+\Delta \mathrm{P}_{\mathrm{j}}+ \\
+\sum \mathrm{a}_{\mathrm{ji}}\left(1.0-\partial_{\mathrm{i}}\right)\left(\Delta \mathrm{Y}_{\mathrm{i}}-\Delta \mathrm{X}_{\mathrm{i}}\right) \\
\sum \mathrm{a}_{\mathrm{ji}}\left(\mathrm{P}_{\text {gi }}-\mathrm{P}_{\text {liav }}-\Delta \mathrm{X}_{\mathrm{i}}+\mathrm{PNS}_{\mathrm{i}}\right) \geq \mathrm{P}_{\mathrm{jmin}}-\Delta \mathrm{P}_{\mathrm{j}}+ \\
+\sum \mathrm{a}_{\mathrm{ji}}\left(1.0-\partial_{\mathrm{i}}\right)\left(\Delta \mathrm{Y}_{\mathrm{i}}-\Delta \mathrm{X}_{\mathrm{i}}\right)
\end{array}
$$

A reinforcement plan (if there is one) will be identified by solving the initial deterministic OPF study followed, for each identified vertex, by the two parametric problems, as before. The second parametric study has to be solved until the value of the parameter $\partial$ 
reaches the $\alpha$-cut corresponding to the specified exposure index. The reinforcement values correspond to maximum values of variables $\Delta \mathrm{P}_{\mathrm{gi}}$ and $\Delta \mathrm{Pj}$.

The target exposure index value may not be achievable for a given set of system components to be reinforced. In fact, the desired value will only be obtained if new iterations of the Dual Simplex Method are performed considering the inclusion of new variables in the problem; however, that may not be possible while keeping feasibility. Therefore, $\Delta \mathrm{P}_{\text {gi }}$ and $\Delta \mathrm{P}_{\mathrm{j}}$ variables play a role similar to the variables related to power not supplied, because they allow keeping mathematical feasibility but display system unfeasibilities.

\begin{tabular}{|c|c|c|c|c|c|c|c|}
\hline bus & $\begin{array}{r}\text { Gen. } \\
\text { no. }\end{array}$ & $\begin{array}{l}\text { Pgmax } \\
\text { (MW) }\end{array}$ & $\begin{array}{l}\text { Inc cost } \\
(\$ / M W h)\end{array}$ & bus & $\begin{array}{r}\text { Gen. } \\
\text { no }\end{array}$ & $\begin{array}{c}\text { Pgmax } \\
(\mathrm{MW}) \\
\end{array}$ & $\begin{array}{l}\text { Inc cost } \\
(\$ / \mathrm{MWh})\end{array}$ \\
\hline 1 & 1 & 40.0 & 3.0 & 15 & 3 & 24.0 & 2.0 \\
\hline 1 & 2 & 40.0 & 3.0 & 15 & 4 & 24.0 & 2.0 \\
\hline 1 & 3 & 152.0 & 4.0 & 15 & 5 & 24.0 & 2.0 \\
\hline 1 & 4 & 152.0 & 4.0 & 15 & 6 & 310.0 & 6.0 \\
\hline 2 & 1 & 40.0 & 3.0 & 16 & 1 & 310.0 & 5.5 \\
\hline 2 & 2 & 40.0 & 3.0 & 18 & 1 & 800.0 & 9.0 \\
\hline 2 & 3 & 152.0 & 4.0 & 21 & 1 & 800.0 & 8.0 \\
\hline 2 & 4 & 152.0 & 4.0 & 22 & 1 & 100.0 & 2.0 \\
\hline 7 & & 200.0 & 5.0 & 22 & 2 & 100.0 & 2.0 \\
\hline 7 & & 200.0 & 5.0 & 22 & 3 & 100.0 & 2.0 \\
\hline 7 & & 200.0 & 5.0 & 22 & 4 & 100.0 & 2.0 \\
\hline 13 & & 394.0 & 6.0 & 22 & 5 & 100.0 & 2.0 \\
\hline 13 & & 394.0 & 6.0 & 22 & 6 & 100.0 & 2.0 \\
\hline 13 & & 394.0 & 6.0 & 23 & 1 & 310.0 & 5.0 \\
\hline 15 & 1 & 24.0 & 2.0 & 23 & 2 & 310.0 & 5.0 \\
\hline 15 & 2 & 24.0 & 2.0 & 23 & 3 & 700.0 & 7.0 \\
\hline
\end{tabular}

Tab. 1 - Generator characteristics.

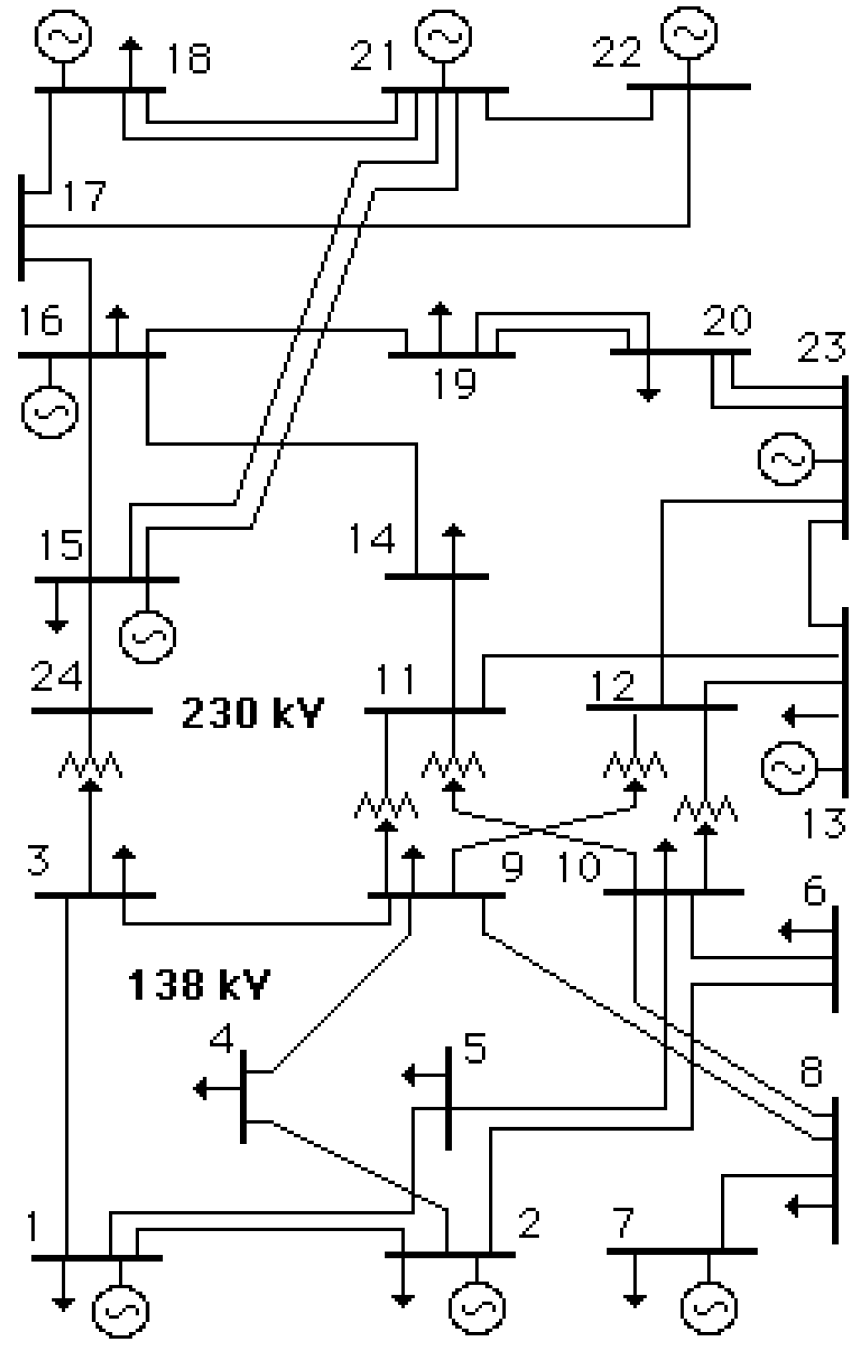

Fig. 7 - IEEE RTS network.

\section{CASE STUDY}

The methodologies described are exemplified using the 24 bus, 38 branch, 32 generator network presented in Fig. 7. This network is based on the IEEE Reliability Test System - RTS (a 500 MVA power base was adopted). Generator characteristics are presented in table 1. The central values of the $1.0 \alpha$-cut of each load distribution correspond to the RTS values multiplied by 1.8 and the original generator limits were doubled while branch data of the RTS remained unchanged.

In this example trapezoidal fuzzy numbers were adopted to represent load uncertainties. The extreme values of the 0.0 and 1.0 $\alpha$-cuts correspond to $(0.9,0.95,1.05,1.1)$ of the central value.

\begin{tabular}{|c|c|c|c|c|c|}
\hline \multirow{2}{*}{\multicolumn{2}{|c|}{ bus Gen. no }} & \multicolumn{4}{|c|}{ Pg possibility distribution (MW) } \\
\hline & & a1 & a2 & a3 & a4 \\
\hline 7 & 1 & 0.0 & 0.0 & 11.25 & 22.50 \\
\hline 7 & 2 & 177.50 & 188.75 & 200.00 & 200.00 \\
\hline 7 & 3 & 200.00 & 200.00 & 200.00 & 200.00 \\
\hline 13 & 1 & 281.92 & 323.42 & 394.00 & 394.00 \\
\hline 18 & 1 & 0.0 & 0.0 & 0.0 & 0.0 \\
\hline 21 & 1 & 0.0 & 149.81 & 375.88 & 612.16 \\
\hline 23 & 1 & 310.00 & 310.00 & 310.00 & 310.00 \\
\hline 23 & 3 & 330.04 & 575.32 & 700.00 & 700.00 \\
\hline \multicolumn{6}{|c|}{ Tab. 2 - Generator possibility distributions (FOPF). } \\
\hline \multirow{2}{*}{\multicolumn{2}{|c|}{ extr. nodes }} & \multicolumn{4}{|c|}{ P possibility distribution (MW) } \\
\hline & & a1 & a2 & a3 & $\mathrm{a} 4$ \\
\hline 1 & 2 & -42.59 & -26.09 & 7.88 & 25.24 \\
\hline
\end{tabular}




\begin{tabular}{|ccrrrr|}
\hline 1 & 5 & 103.20 & 116.49 & 143.06 & 156.91 \\
2 & 6 & 82.19 & 92.74 & 113.84 & 124.77 \\
5 & 10 & -26.86 & -12.44 & 16.40 & 31.42 \\
8 & 10 & -69.29 & -63.33 & -51.41 & -45.00 \\
12 & 23 & -342.80 & -317.04 & -247.80 & -191.32 \\
13 & 23 & -197.04 & -172.22 & -97.13 & -36.82 \\
20 & 23 & -477.48 & -463.16 & -415.36 & -360.94 \\
\hline
\end{tabular}

Tab. 3 - Power flow possibility distributions (FOPF).

\begin{tabular}{|crrrrr|}
\hline bus & Gen. no. & \multicolumn{4}{c|}{ Pg possibility distribution (MW) } \\
a1 & a2 & a3 & a4 \\
\hline \hline 7 & 1 & 0.0 & 0.0 & 11.25 & 22.50 \\
7 & 2 & 177.50 & 188.75 & 200.00 & 200.00 \\
7 & 3 & 200.00 & 200.00 & 200.00 & 200.00 \\
13 & 1 & 299.71 & 332.32 & 394.00 & 394.00 \\
18 & 1 & 0.0 & 0.0 & 0.0 & 0.0 \\
21 & 1 & 0.0 & 22.15 & 275.32 & 400.98 \\
23 & 1 & 310.00 & 310.00 & 310.00 & 310.00 \\
23 & 3 & 548.58 & 700.00 & 700.00 & 700.00 \\
\hline
\end{tabular}

Tab. 4 - Generator possibility distributions (random sampling).

\begin{tabular}{|ccrrrr|}
\hline extr. nodes & \multicolumn{5}{c|}{ P possibility distribution (MW) } \\
a1 & a2 & a3 & a4 \\
\hline \hline 1 & 2 & -32.58 & -20.89 & 3.59 & 16.39 \\
1 & 5 & 111.15 & 120.46 & 140.14 & 150.50 \\
2 & 6 & 88.80 & 96.04 & 111.82 & 120.36 \\
5 & 10 & -18.90 & -8.46 & 12.53 & 23.02 \\
8 & 10 & -78.34 & -67.93 & -46.72 & -36.08 \\
12 & 23 & -324.69 & -307.98 & -274.23 & -241.08 \\
13 & 23 & -180.52 & -163.96 & -132.28 & -91.88 \\
20 & 23 & -472.81 & -456.74 & -424.11 & -407.55 \\
\hline
\end{tabular}

Tab. 5 - Power flow possibility distributions (random sampling).

\section{Base case}

The FOPF algorithm was used to study the base case corresponding to the operation of all system components. In tables 2 and 3 some of the calculated generated active power and branch possibility distributions are presented. Values a1 and a4 correspond to the extreme values of the 0.0 -cut and a 2 and a 3 to the 1.0 -cut. The performance of the proposed algorithm was evaluated by assigning an uniform probability distribution to the 0.0 and 1.0-cut intervals of each specified load powers. This way, for 0.0 and 1.0-cuts 5000 deterministic load scenarios were randomly sampled and solved with a deterministic DC OPF algorithm. The extreme values obtained for each generated power and branch flow were used to rebuild an approximation to their distributions. In tables 4 and 5 examples of such distributions are present. The bad (over optimistic) results obtained with the Monte Carlo type simulation can be readily noticed: the interpretation of this fact will be given below.

\section{Line 3-24 outage}

System operation was also studied considering branch 3-24 outage. In this case, the system has no capacity to accommodate the load related to 1.0 -cut. In figure 8 the respective PNS possibility distribution (curve PNS1), obtained with a single FOPF exercise, is sketched. The approximate possibility distribution obtained by a random sampling process is also represented (curve PNS2). This distribution was built by evaluating the extreme values of the $0.0,0.25$, $0.5,0.75$ and 1.0 -cuts. For each $\alpha$-cut, an uniform probabilistic distribution was associated to each load uncertainty so that 5000 load scenarios were sampled and analyzed with a deterministic DC OPF formulation, involving the global effort of solving 25000 deterministic problems. Curve PNS1 reveals a nonlinearity at point $\mathrm{P}$ due to the adoption of the fuzzy union to aggregate the partial results obtained from each parametric OPF study. As detailed in figure 8 , point $\mathrm{P}$ is the intersection point of the PNS curves obtained from two of these parametric studies.

The analysis of PNS1 and PNS2 possibility distributions demonstrate that the random sampling process, although associated with a much heavier computational burden, leads to incorrect PNS distributions. This occurs as one is interested in "what may happen", and so extreme cases that by chance were not sampled have a definite influence in the shape of the possibility distributions. Therefore, Monte Carlo type techniques are not suited to the kind of assessments one is aiming at.

In figure 9, one displays the values of generating capacity reinforcements, needed to obtain decreasing exposure indices (Iexp). There is no way of reaching an exposure index lower than .38, no matter how much one is willing to pay in generating capacity in buses 1 and 2 . In figure 8 , the PNS possibility distributions obtained using the FOPF algorithm for each of the above mentioned reinforcements (230.36 MW or $259.18 \mathrm{MW}$ ) are also sketched in curve PNS3. They coincide, suggesting that the same bottlenecks are constraining the system for exposure values below 0.38 .

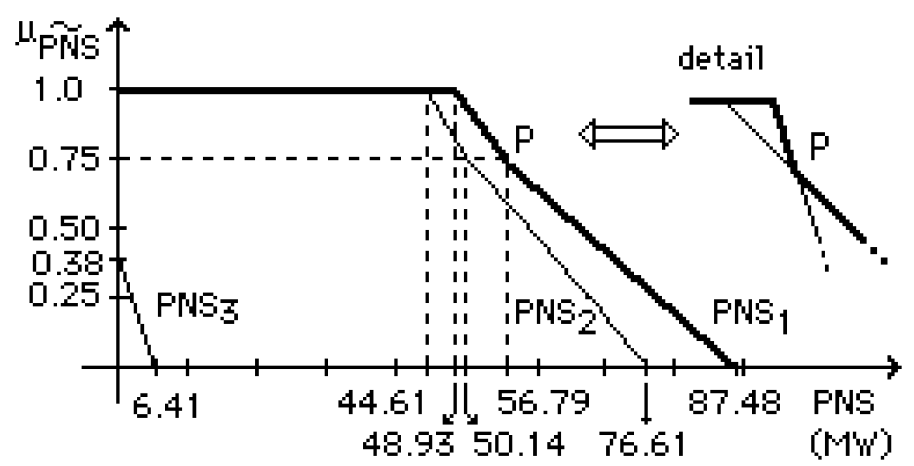

Fig. 8 - PNS if branch 3-24 is out of service (see text).

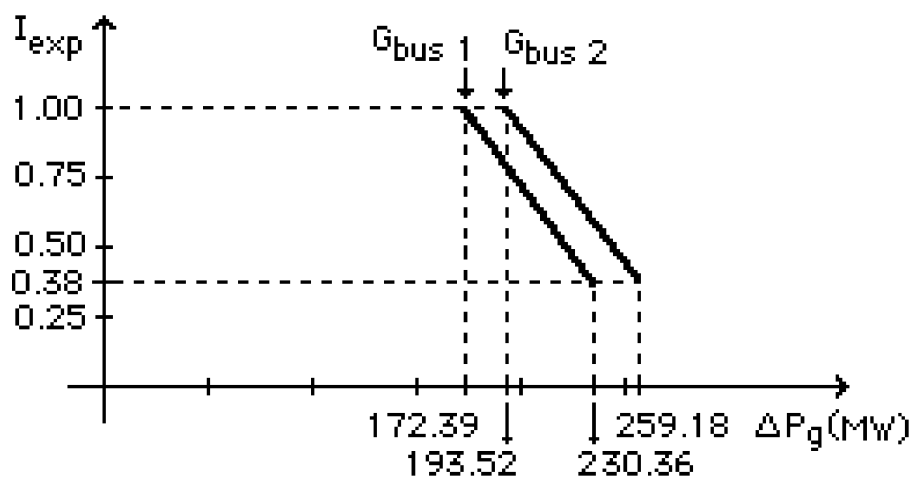

Fig. 9 - Evolution of exposure ind. for two reinforcement strategies.

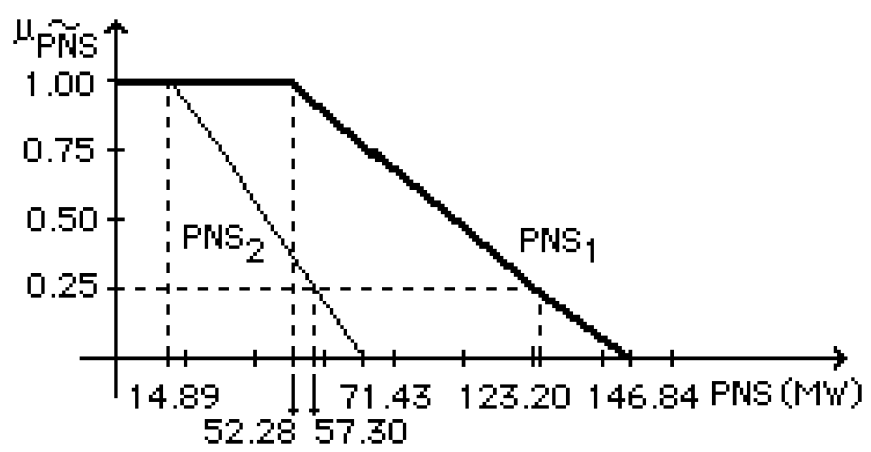

Fig. 10 - PNS if generators $7 / 3,13 / 1$ and $23 / 2$ are out of service. 


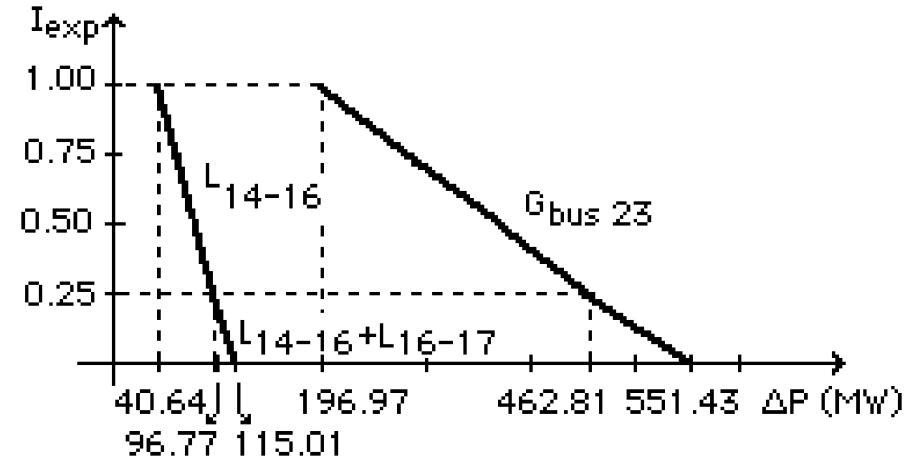

Fig. 11 - Evolution of exposure ind. for two reinforcement strategies

\section{Generators $7 / 3,13 / 1$ and 23/2 overlapping outages}

In this case the system exposure and robustness indices are 1.0 and 0.0. The PNS possibility distributions resulting from the FOPF algorithm (curve PNS1) and from a random sampling process (curve PNS2) are sketched in figure 10. There is here an obvious deviation of the random sampling results from those (more "exact") obtained with the FOPF model.

Reinforcement plans for branch 14-16 and bus 23 are presented in figure 11. The increase in branch 14-16power flow limit is not enough to reduce exposure to 0.0 , due bottlenecks imposed by other system components: the detection of these components is easily made by inspection of the Simplex results and the binding constraints. But if reinforcements in branches 14-16 and 16-17 (by 114.15 and 0.87 MW) are admitted then a totally robust solution can be reached.

These results show that a good evaluation of the risk associated to system design can be obtained from a FOPF algorithm. Moreover, a FOPF demands solving only one deterministic optimal power flow plus in general a reduced number of parametric problems: on average, this takes 1.4 of the time for just a deterministic OPF. Therefore, the efficiency of a FOPF approach when compared to the random sampling of load scenarios is impressive.

\section{CONCLUSIONS}

The fuzzy modelling of load (and generation) uncertainties allows the planner to have a new perspective in assessing the merits of alternative system designs: he can now have readily available useful information on the risks derived from planning decisions.

Therefore, he can evaluate not only the degree of flexibility of each alternative, in terms of possible futures for which a particular plan is acceptable, but also how much does it cost to increase the robustness of each solution: and he has a means of comparing alternatives in equal risk basis.

With the described FOPF approach, a high degree of computer efficiency is reached. One does not know a priori which are the most severe load scenarios in a power system: it has been shown $[4,5]$ that, for many system components, the worst scenario is not the one with all the loads at their highest value. Therefore, an alternative to the FOPF would be a Monte Carlo type sampling of load scenarios, heavy and costly in computing terms.

Moreover, as a very important result, it has been demonstrated that a Monte Carlo random sampling strategy, in order to try to assess the influence of uncertain load forecasts in system robustness, may lead in general to an incorrect over-optimistic picture of the flexibility of system design.
In this paper, the problem of combining fuzzy representation of generations and loads with probabilistic events such as generator or branch outages has not been addressed. However, this subject has already been dealt with [9] and will be the object of further publication. In fact, fuzzy and probabilistic representations must be seen as complementary and not as competitive, because they deal with different kinds of uncertainties.

\section{REFERENCES}

[1] L. A. Zadeh, "Fuzzy Sets", Information and Control, pp. 338 353, August 1965.

[2] D. Dubois, H. Prade, Theorie des Possibilités - Application à la representation des connaissances en informatique, $2^{\mathrm{a}}$ Edition, Masson, Paris, 1987.

[3] V. Miranda, M. A. Matos, "Distribution System Planning with Fuzzy Models and Techniques", Proceedings of CIRED 1989, Brighton, August 1989.

[4] V. Miranda, M. A. Matos, J. T. Saraiva, "Fuzzy Load Flow - New Algorithms Incorporating Uncertain Generation and Load Representation", 10th PSCC, Graz, August 1990; in Proceedings of the 10th PSCC, Butterworths, London, 1990.

[5] J. T. Saraiva, V. Miranda, M. A. Matos, "Generation and Load Uncertainties Incorporated in Load Flow Studies", proceedings of MELECON 91 (IEEE s.8), Ljubljana, May, 1991.

[6] V. Miranda, J. T. Saraiva, "Fuzzy Modelling of Power System Optimal Load Flow", IEEE Trans. Power Systems, Vol. 7, No. 2, May 1992.

[7] T. Gal, Post- Optimal Analysis, Parametric Programming and Related Topics, McGraw Hill, New York, 1979.

[8] H. M. Merril, A. J. Wood, "Risk and Uncertainty in Power System Planning", presented at the 10th PSCC, Graz, Austria, August 1990.

[9] V. Miranda, L. M.V.G. Pinto, "Um modelo para a consideração de incertezas na operação de Sistemas Elétricos", SNPTEE Seminário Nacional de Produção, Transmissão e Distribuição de Energia Eléctrica, Brasil, Out.91

\section{Acknowledgment}

The research work reported is being done under a project partially sponsored by contract PMCT/C/TIT/413/90 of JNICT.

João Tomé Saraiva was born in Oporto, Portugal, on August 18, 1962. He received his licenciate, an M.S. equivalent and Ph.D. degrees from FEUP in 1985, 1988 and 1993, respectively, all in electrical engineering. In 1985 he joined FEUP and holds currently the position of Auxiliar Professor. In 1989 he joined also INESC as a researcher.

Vladimiro Miranda was born in Oporto, Portugal, on March 11, 1955. He received his licenciate and Ph.D. degrees from the Faculty of Eng. of the University of Oporto (FEUP) in 1977 and 1982, respectively, all in electrical engineering. In 1981 he joined FEUP and currently holds the position of Associate Professor. In 1985 he joined also INESC - a Research Institute in Systems and Computer Engineering - and holds currently the position of Project Manager - Head of Information and Decision in Energy Systems Area.

Leontina Maria V. Graziadio Pinto was born in Coimbra, Portugal in 1958. She received her Bsc in Electrical Engineering from PUC/RJ in 1979, and MSc and DCs degrees from COPPE/UFRJ and IM/UFRJ in 1980 and 1990 respectively. She is now with the Catholic University of Rio de Janeiro (PUC/RJ); her current interests are research and education in Planning and Operation of Electric Systems. 